\title{
Das Rindal; zur Genese eines Urstromtales in der NE-Schweiz
}

\section{Die Bedeutung der Urstromtäler für das glaziale Geschehen}

Im Stirnbereich eiszeitlicher Gletscher bestimmen Moränenwälle, Zungenbecken und Schotterfelder das Bild. Entlang des seitlichen Eisrandes, wo die Gletscher durch relativ steile Bergflanken klare Begrenzungen erhielten, wird die Eishöhe vermehrt durch Schmelzwasserwege angezeigt. Sie liegen oft treppenartig übereinander oder enthalten bei langfristiger Entwicklung in sich selbst verschiedene Phasen.

TROLL (1936) sowie MAAG (1967) betonen besonders die Bedeutung der randglazialen Entwässerungswege: Sie können nur bei längerem Verweilen des Gletschers in der betreffenden Höhenlage entstehen, denn nur dann werden die Schmelzwässer in solche Rinnen gezwungen. Sie markieren somit andauernde Eisrandlagen.

Urstromtäler lassen in ihrer Lage das Niveau des jeweiligen intraglazialen Wasserspiegels erkennen und dokumentieren damit in etwa die Höhe des Gletscherrandes. In Verbindung mit Moränenwällen und Schotterfeldern dienen sie der Festlegung und Korrelation eiszeitlicher Gletscherstände. Ihr häufiges Auftreten widerspiegelt die Bedeutung der Glaziale für die morphologische Ausgestaltung der Molasse-Bergländer. Das Rindal ist ein typisches Beispiel eines voralpinen Urstromtales. An seiner Entwicklung können die Beziehungen zwischen dem phasenweisen Geschehen an den Eisfronten und in den Schmelzwassertälern besonders klar aufgezeigt werden.

\section{Zur Lage des Rindales}

Abrupt und markant in die sanftwelligen nw Ausläufer der Hochzone des Degersheimer Berglandes mit Höhen über $800 \mathrm{~m}$ eingeschnitten, steht das Rindal als $200 \mathrm{~m}$ tiefe und $7 \mathrm{~km}$ lange Kerbe in auffälligem Gegensatz zur Morphologie seiner Umgebung (Fig. 1 und 2). Seit dem Hochmittelalter verbindet diese Talung als wichtige Verkehrsader ohne nennenswerte Höhendifferenzen St. Gallen über Flawil mit dem mittleren Toggenburg. Das heutige Trockental ist mit seinen weiten Mäandern und dem flachen Talboden das ausgeprägteste Urstromtal e der Thur.

Dr. phil. O. Keller, Rorschacherstraße 44, 9000 St. Gallen

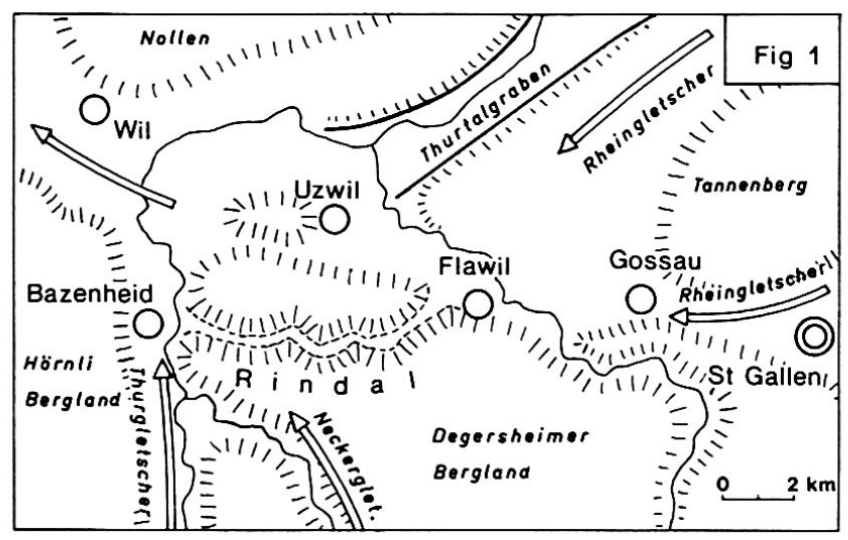

\section{Die präwürmzeitliche Landschaft}

Die präwürme Landoberfläche des Rindalgebietes (Fig. 2,3) läßt sich in Anlehnung an die sanften Formen der Hügel und Mulden auf der S-Seite sowie einzelner Höhen im $\mathrm{N}$ einigermaßen rekonstruieren. Diese Hochzonen wurden nur im äußersten WürmMaximum während kurzer Zeit vom Eis modelliert. Die Entwässerung erfolgte vor dem letzten Glazial großenteils nach W. Der Zusammenfluß zweier bedeutender Bäche im Raume Oberrindal zeichnete, in die Tiefe projiziert, das heutige Talknie vor. Gegen eine n-wärts gerichtete Entwässerung in diesem Bereich (SAXER, 1969) spricht die Höhenlage der entsprechenden Paßlücken. Hingegen macht im Ostabschnitt die weite, moränenbedeckte Lücke bei Eppenberg mit tieferem Niveau einen nach $\mathrm{N}$ gerichteten Bachlauf wahrscheinlich. Ähnlich wurde auch die E-Pforte des Rindals durch ein n-wärts fließendes Gewässer vorgezeichnet. Zwischen den einzelnen Bachsystemen scheinen flache Paßübergänge um $750 \mathrm{~m}$ in E-W-Richtung die Anlage des Rindals beeinflußt zu haben.

\section{Anlagen vor und während dem Würm-Maximum}

Erratiker bis auf die höchsten Kuppen beidseits des Rindals sowie der Vergleich mit Eisrandlagen w der Thur (ANDRESEN, 1964) und im Neckertal (KELLER, 1973) zeigen, dass im Würm-Maximum das Eis die ganze Region bis in eine Höhe zwischen 850 und $900 \mathrm{~m}$ überflossen hat. Die E-W gerichtete Eisbewe- 


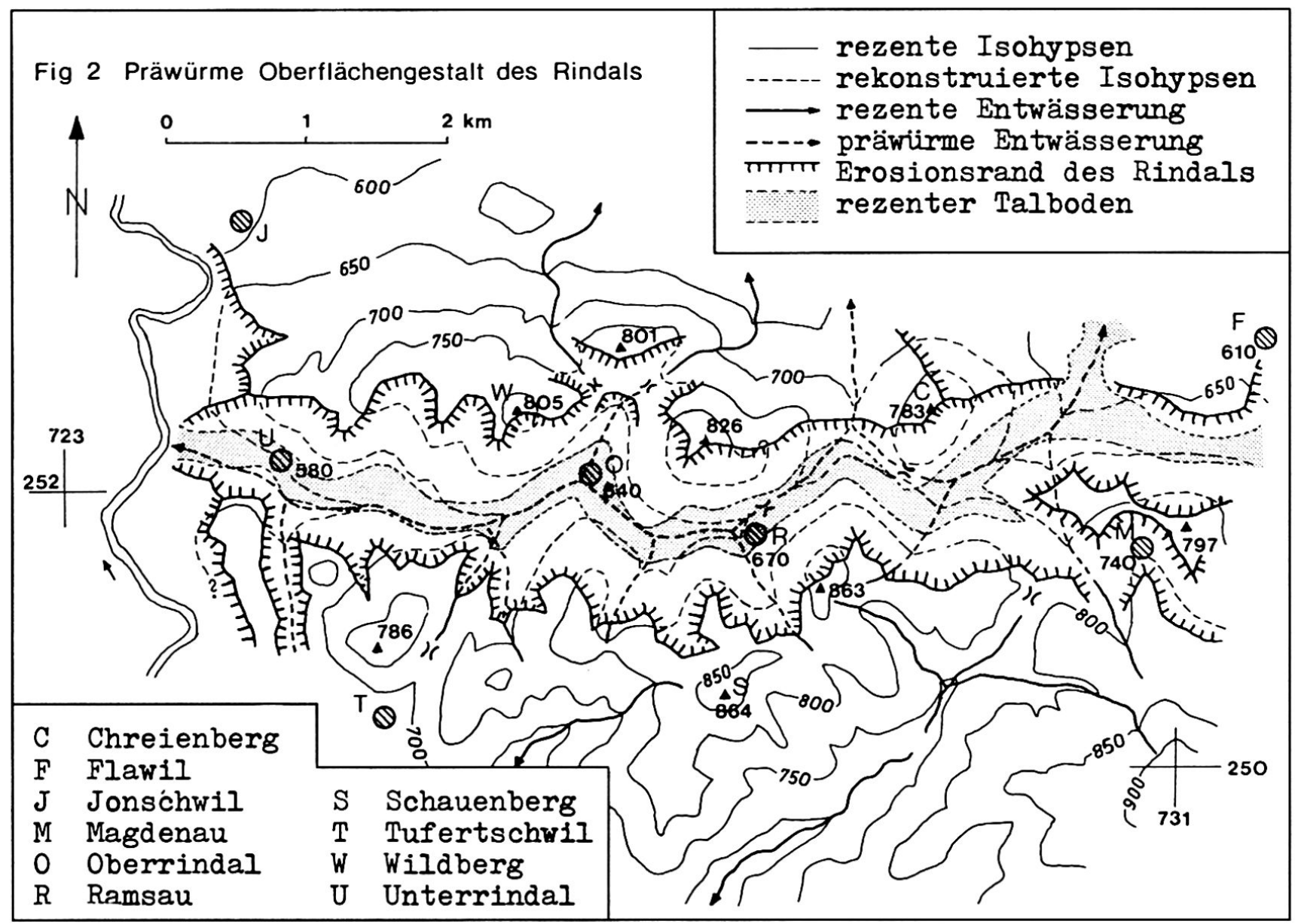

gung des vorstoßenden Bodensee-Rheingletschers begünstigte die Auskolkung der ebenso angeordneten Paßübergänge im Bereiche der Rindaler Rinne.

Mit der nach dem Würm-Maximum einsetzenden Absenkung der Eisoberfläche hielt die Tieferlegung der Pässe über dem heutigen Rindal Schritt durch die Tätigkeit von Eis und subglazialen Wässern. Damit wurde die so vorgezeichnete Rinne langsam umgestaltet und in den folgenden Stadien zum Schmelzwasserweg weiterentwickelt.

Einige Besonderheiten machen es möglich zu zeigen, $\mathrm{da} ß$ die Ausgestaltung des Rindals im wesentlichen erst im letzten Glazial und zwar vorwiegend in den Stadien nach dem Würm-Maximum erfolgte:

1. Die rezenten Formen des Rindals sind fluvial, markant und unüberprägt. Bei Annahme rißzeitlichen Alters wären diese verwischt und ausgeglichen wor- den. Dabei darf allerdings eine hochgelegene Rißanlage mit Vorzeichnung der großen Linien nicht ausgeschlossen werden.

2. Erst im Würm-Maximum stiegen die Eismassen wieder zu derjenigen Höhe an, die nötig war, daß Ausgestaltung und Tieferlegung der Rindaler Rinne überhaupt einsetzen konnten.

3. Fig. 3: Die von einer Eisrandlage ausgehende, zum Rindal weisende Schotterfläche von Eberwis endet abrupt $70 \mathrm{~m}$ über dem Tal. Das Zungenbecken mit Seitenmoränen eines Thurgletscherlappens von Tufertschwil her liegt $80 \mathrm{~m}$ über dem Rindal auf einer Hochfläche, wobei der Stirnbereich, der über der heutigen Talung lag, wegerodiert ist. Das gefällsarme, hochgelegene und mit Mäandern ausgestattete Schmelzwassertal von Magdenau führt mit einer engen, V-förmigen Steile ins Rindal hinunter. Diese drei Bildungen 


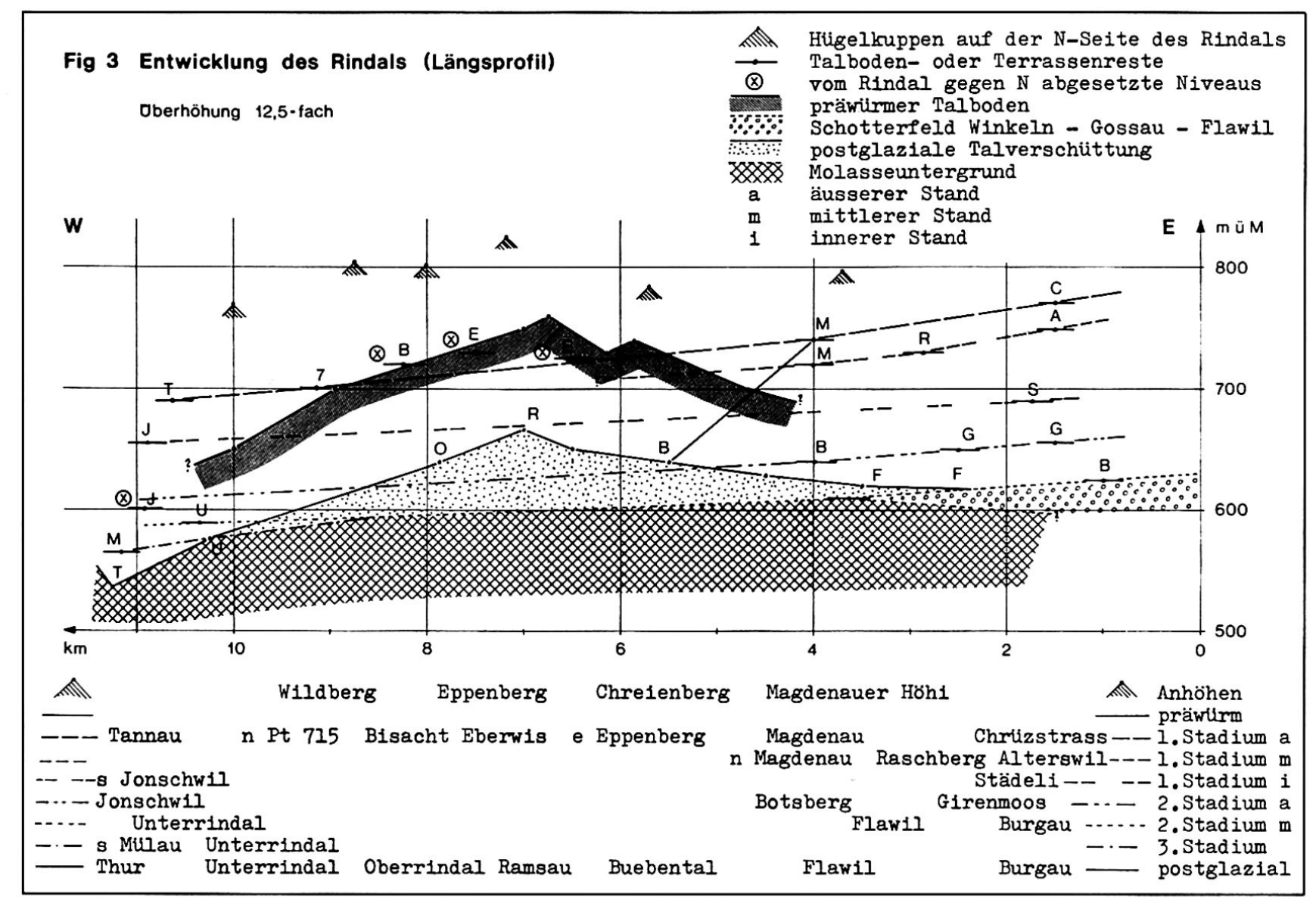

weisen das tiefer liegende Rindal als jünger aus, weshalb seine Ausgestaltung in die internen und hier weniger hohen Stadien nach dem Würm-Maximum zu stellen ist.

\section{Die Entwicklung im 1. Stadium nach dem Würm-Maximum}

Diesem Stadium entsprechen beim Rheingletscher Eisrandlagen im Raume Dießenhofen und bei Alten (Thurtal) oder aber Schlieren beim Linthgletscher. Zwischen Herisau und Wil können 3 Stände des Rheingletschers durch Wallmoränen, Schotterfluren und Schmelzwasserwege unterschieden werden.

Im äußeren Stand (Fig. 3, 4) wurde die Rinne Degersheim-Wolfertswil-Magdenau angelegt. Der Rheingletscher stand $\mathrm{n}$ des Rindals bei Eberwis (Schotterfeld gegen SW zum Rindal) und bei Bisacht (Schmelz- wasserrinne nach S). Im Winkel der Konfluenz von Rhein- und Thurgletscher wurde s Jonschwil die Wallmoräne von Hori mit der Schotterflur von Tannau akkumuliert. Von Tufertschwil stieß der Thurgletscher mit einem Lappen über Pt. 715 nach $\mathrm{N}$ und legte Zungenbecken und Seitenmoränen auf der Hochfläche über dem heutigen Tal an. Aus diesen erhaltenen Resten läßt sich die damalige Höhenlage des Rindals rekonstruieren.

Während den folgenden Rückzügen der Gletscher vermochte die Tiefenerosion im Rindal mit der Absenkung der Eisoberfläche Schritt zu halten, während die Magdenauer Rinne und die Durchgänge Eberwis, Bisacht und $\mathrm{n}$ Tufertschwil trocken fielen. Die Erklärung liegt im hohen Schmelzwasseranfall zum Rindal, welches alles Wasser aus dem Raume Herisau-Gossau -Flawil aufnehmen mußte, während die anderen Rinnen nur lokale Entwässerungsfunktion hatten. 


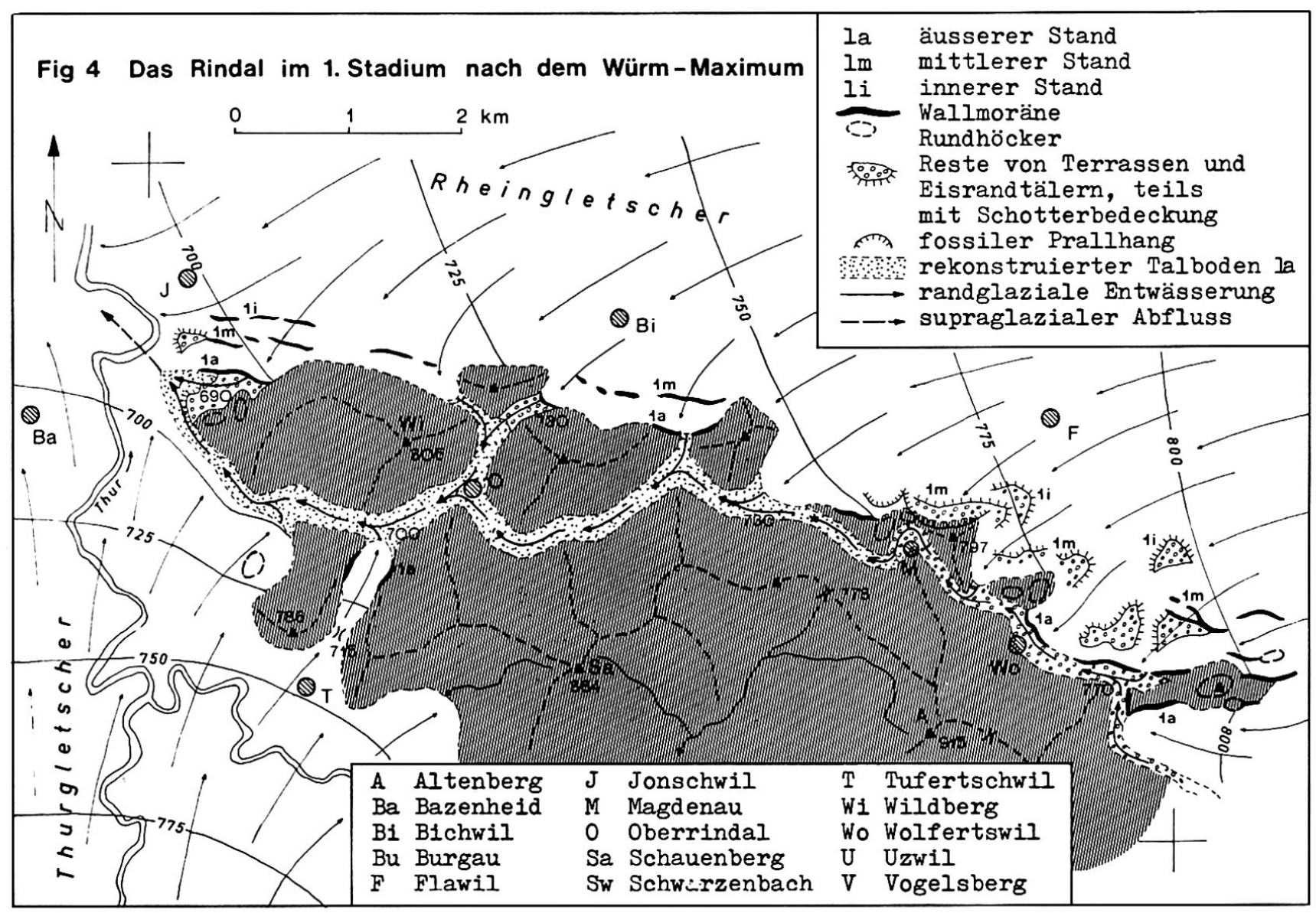

Der mittlere Stand (Fig. 3, 4) schuf ein um ca. $20 \mathrm{~m}$ tieferes Niveau, wie Talboden- und Mäanderreste $n$ Magdenau zeigen.

Während dem inneren Stand (Fig. 3, 4) wurde im E der Rindaler Rinne neu die Glatt mit einbezogen und daher hier der Talboden stärker tiefergelegt als im W, wo sich die Entwässerung auf das Niveau der KamesSchotter s Jonschwil in $655 \mathrm{~m}$ einpendelte. Da sich Rhein- und Thurgletscher noch berührten, erfolgte der weitere Abfluß supra- oder intraglazial Richtung $\mathrm{W}$.

\section{Das 2. Stadium nach dem Würm-Maximum}

Der Rheingletscher stirnte im Raume Stein a/Rh. einerseits und Andelfingen andererseits. Dieses Stadium wird mit «Zürich» des Linthgletschers parallelisiert.
Zwischen Gossau und Wil können 3 Hauptstände auseinandergehalten werden, welche auch der Thurgletscher im Raume Bazenheid aufweist (FALKNER, 1909; HANTKE, 1961; KELLER, 1973).

Der Eisrand ist im äußern Stand (Fig. 3, 5) durch Moränenwälle s Gossau-Flawil belegt. Das Schmelzwasser und die Glatt suchten den Weg entlang der Eisbegrenzung nach $\mathrm{W}$, wobei die Burgau-BotsbergRinne auf das rezente Niveau abgesenkt wurde. Wallmoränen am Chreienberg zeigen, da $\beta$ als Entwässerungsweg nach $\mathrm{W}$ das Rindal benutzt und dabei um weitere $50 \mathrm{~m}$ gegenüber dem inneren Stand des 1. Stadiums tiefergeschaltet wurde.

Das Rindal dürfte im Querschnitt der Burgau-Rinne entsprochen haben, da diese wegen der anschließenden Trockenlegung als Maßstab genommen werden kann. Der Schmelzwasseranfall muß darnach wesentlich größer als in den früheren Stadien gewesen sein, 


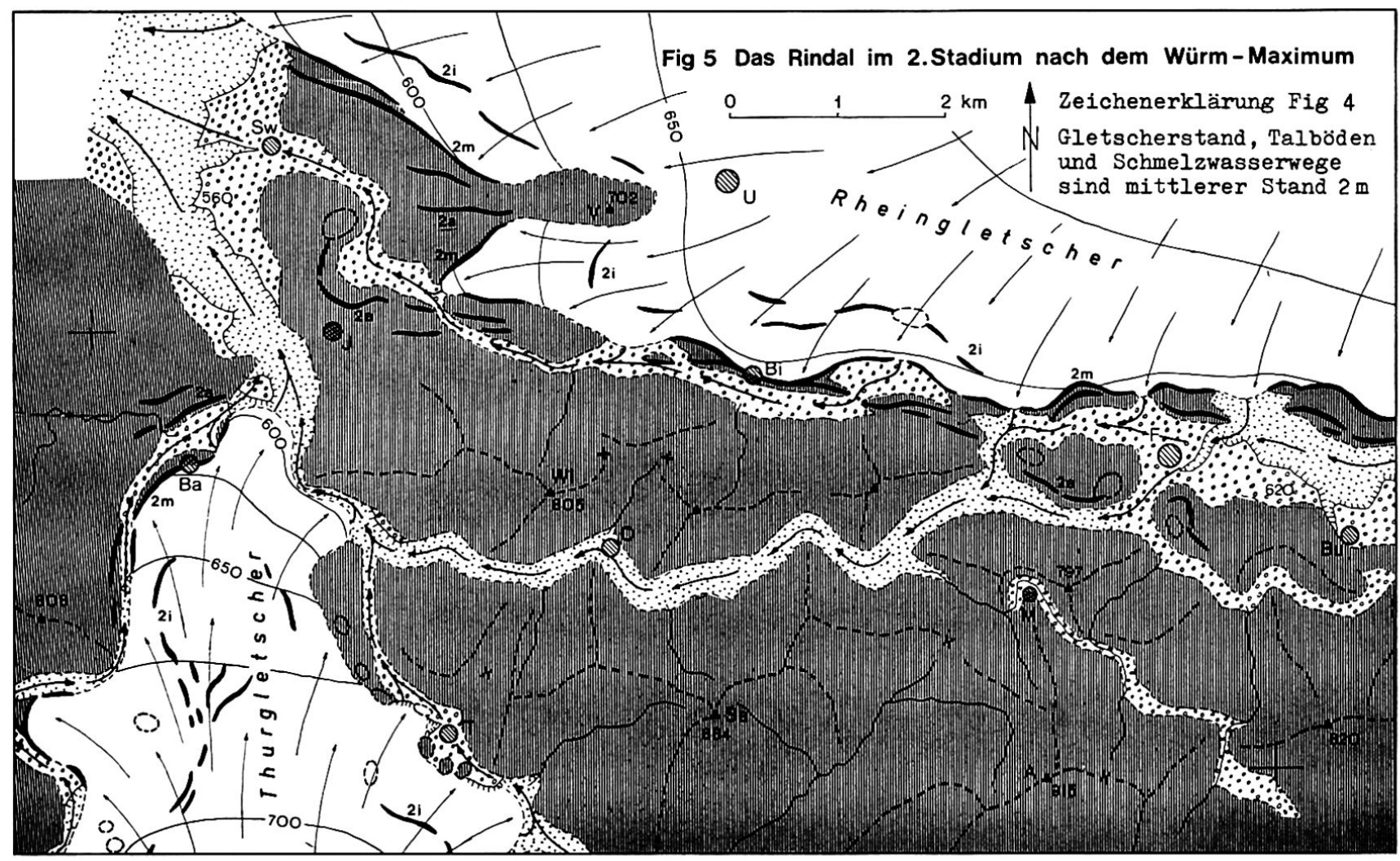

erreichte aber noch nicht die Ausmaße des folgenden Standes.

Der äußerst markante mittlere Stand (Fig. 3, 5) zeichnet sich durch mehrere Staffeln hoher Wallmoränen aus, die sich von Gossau über Flawil bis Uzwil verfolgen lassen. Es zeigt sich, daß der Rheingletscher auf dem Gossauer Plateau zwar etwas geringere Ausdehnung als im äußern Stand hatte, aber nochmals beinahe dieselbe Eishöhe erreichte. W Flawil bildeten beide Stände bei Bichwil sogar eine Einheit. Dieses Zusammenfallen bewirkte, daß das Schmelzwasser wiederum ins Rindal gezwungen wurde, wobei e davon die mächtige Schotterflur von Winkeln (St. Gallen)-Gossau-Flawil geschüttet wurde. Das Niveau von $610 \mathrm{~m}$ bei Flawil entspricht dem Talboden am Eingang zum Rindal. Der Ausgang hingegen lag, verglichen mit dem Feld von Schwarzenbach, auf ca. $590 \mathrm{~m}$. Dieses Niveau ist bei Unterrindal in Form einer Terrasse am $n$ Talhang erhalten.

In dieser Phase wurde das Rindal zur heutigen Größe ausgeweitet, woraus auf gewaltige Schmelzwassermen- gen geschlossen werden kann. Die gesamte Eisrandentwässerung von St. Gallen her und aus dem Appenzellerland erfolgte denn auch durch diesen Talzug.

Im innern Stand (Fig. 5) wurde die Höhe der Chreienbergausläufer nicht mehr erreicht, weshalb das Schmelzwasser direkt dem Eisrand entlang nach W abfließen konnte und das Rindal als randglazialer Weg endgültig ausgeschaltet wurde.

\section{Die spät- und postglaziale Umgestaltung}

Das nur noch lokale Wasser des Rindals paßte vorerst seinen Lauf am W-Ende durch Bildung eines etwas tieferen Talbodens bei Unterrindal dem entsprechenden Thur-Niveau des 3. Stadiums (Konstanz/ Hurden) an, welches durch Schotterfluren und Terrassen vom Eisrand bei Kradolf bis gegen Lichtensteig verfolgt werden kann (ANDRESEN. 1964; KELLER, 1973). Mit der anschließenden Eintiefung des Thur- 
laufes auf das rezente Niveau wurde dieser Talboden w Unterrindal nochmals zerschnitten.

Nach dem Wegfall des Schmelzwasserstromes im Rindal hörte auch der Abtransport des lokalen Schuttes von den übersteilten Hängen auf. Kleine Seitenbäche legten erosive Runsen in den Talflanken an, während die Sohle durch Schuttkegel eingedeckt wurde. Die Aufschüttung war im Bereiche der größten Eintiefung am stärksten, so daß seither ab Ramsau die Entwässerung rückläufig gegen Flawil erfolgt. Die Talwasserscheide liegt heute bei $670 \mathrm{~m}$, womit hier mit $70 \mathrm{~m}$ Talfüllung zu rechnen ist. Bohrungen liegen leider keine vor, aber der Vergleich mit der viel kleineren Rinne von Herisau Bahnhof, wo gegen $30 \mathrm{~m}$ Hangschutt und Geröll nachgewiesen sind (mdl. Mitteilung von Ing. E. Krayss, St. Gallen), zeigt, daß heute inaktive Schmelzwassertäler nachträglich oft kräftig überfüllt worden sind.

\section{Zusammenfassung}

Das heutige Trockental von Rindal wurde durch den Rheingletscher während dem Würm-Maximum durch glaziale Paßerniedrigung unter Mithilfe von subglazialen Wässern vorgeprägt. Im 1. Stadium (Dießenhofen, Alten) war es zur Hauptsache eisfrei und funktionierte als randglaziale Entwässerungsrinne aus dem Raume Degersheim über Magdenau gegen W zum Thurtal. Später floß zusätzlich auch die Glatt durch diese Rinne $a b$, wobei sich s Flawil über BurgauBotsberg ein weiteres markantes Schmelzwassertal entwickelte. Wegen des stets bedeutenden Wasseranfalles vermochte die erosive Tieferlegung des Rindaler Talbodens mit dem stufenweisen Absinken der Eishöhe des Rheingletschers bis in den mittleren Stand des 2. Stadiums (Stein a/Rh.) Schritt zu halten. Die Hauptausgestaltung erfuhr das Tal in dieser Phase, als auch die Schmelzwässer aus dem Appenzellerland und vom St. Galler Rheingletscherlappen über Gossau-Flawil diesen Weg nahmen. Mit dem Rückzug des Gletschers zum inneren Stand des 2. Stadiums wurde das Rindal trocken gelegt, denn die Schmelzwässer konnten jetzt direkt dem Eisrand entlang nach W abfließen. Damit setzte die Zuschüttung des Tales von den übersteilten Hängen her ein.

\section{Literatur und Karten}

ANDRESEN, H., 1964: Beiträge zur Geomorphologie des östlichen Hörnliberglandes; Jb. der St. Gall. Naturw. Ges., 78.

BUECHI, U. P., 1950: Zur Geologie und Paläogeographie der südlichen mittelländischen Molasse zwischen Toggenburg und Rheintal; Diss. Univ. Zürich.

FALKNER, C., 1909: Die südlichen Rheingletscherzungen von St. Gallen bis Aadorf; Jb. der St. Gall. Naturw. Ges. FREY, A. P., 1916: Die Vergletscherung des oberen Thurgebietes; Jb. der St. Gall. Naturw. Ges., 54.

HANTKE, R., 1961: Die Nordostschweiz zur Würmeiszeit; Ecl. Geol. Helv., 54.

KELLER, O., 1973: Untersuchungen zur Glazialmorphologie des Neckertales (Nordostschweizer Voralpen); Diss. Univ. Zürich.

MAAG, H. U., 1967: Ice-dammed lakes and marginal glacial drainage on Axel Heiberg Island; Diss. Univ. Zürich.

SAXER, F., 1968: Erläuterungen zur Grundwasserkarte des Kt. St. Gallen; Jb. der St. Gall. Naturw. Ges., 79. SCHINDLER, C. et al. 1970: Erläuterungen z. Geol. Atlas der Schweiz, Blatt 1093 Hörnli; Schweizer. Geol. Komm. TROLL, K., 1936: Die sogenannte Vorrückungsphase der Würmeiszeit und der Eiszerfall bei ihrem Rückzug; Mitt. der Geogr. Ges. in München, 29.

BUECHI, U. P., 1950: Geologische Karte der südlichen mittelländischen Molasse 1:50000; in: Zur Geol. und Paläogeogr. der s mittelländ. Molasse zw. Toggenburg und Rheintal; Diss. Univ. Zürich.

JAECKLI, H., 1970: Die Schweiz zur letzten Eiszeit 1:550 000; Atlas der Schweiz.

LUDWIG, A., 1930: Blätter 218-221 Flawil-Schwellbrunn 1:25000; Geol. Atlas der Schweiz; Schweizer. Geol. Komm.

NABHOLZ, w. et al. 1970: Blatt 1093 Hörnli 1:25000; Geol. Atlas der Schweiz; Schweizer. Geol. Komm.

SAXER, F., 1968: Grundwasserkarte des Kt. St. Gallen; Jb. der St. Gall. Naturw. Ges., 79.

Landeskarte der Schweiz 1:100 000; Blätter: 28 Bodensee, 33 Toggenburg.

Landeskarte der Schweiz 1:25000; Blätter: 1073 Wil, 1074 Bischofszell, 1093 Hörnli, 1094 Degersheim. 